\title{
Prognostic Value of SYNTAX Score II in Patients with Acute Coronary Syndromes Referred for Invasive Management: A Subanalysis from the SPUM and COMFORTABLE AMI Cohorts
}

\author{
Slayman Obeid $\left(\mathbb{D},{ }^{1}\right.$ Antonio H. Frangieh, ${ }^{1}$ Lorenz Räber, ${ }^{2}$ Nooraldaem Yousif, ${ }^{1}$ \\ Thomas Gilhofer, ${ }^{1}$ Kyohei Yamaji, ${ }^{2}$ Milosz Jaguszewski, ${ }^{1}$ Soheila Aghlmandi, ${ }^{3}$ James Adams, ${ }^{1}$ \\ Yannik Bockhorn, ${ }^{1}$ Christian Templin, ${ }^{1}$ Barbara E. Stähli, ${ }^{1}$ Peter Jüni, ${ }^{3}$ Nicolas Rodondi, ${ }^{4,5}$ \\ François Mach (iD, ${ }^{6}$ Marco Roffi, ${ }^{6}$ Stephan Windecker, ${ }^{2}$ Willibald Maier, ${ }^{1}$ Fabian Nietlispach, ${ }^{1}$ \\ Christian M. Matter, ${ }^{1}$ Roland Klingenberg, ${ }^{1}$ and Thomas F. Lüscher ${ }^{7}{ }^{7}$ \\ ${ }^{1}$ University Heart Center, Department of Cardiology, Zurich, Switzerland \\ ${ }^{2}$ Cardiovascular Center, Department of Cardiology, University Hospital Bern, Bern, Switzerland \\ ${ }^{3}$ Institute of Social and Preventive Medicine and Clinical Trials Unit, Department of Clinical Research, University of Bern, \\ Bern, Switzerland \\ ${ }^{4}$ Department of General Internal Medicine, University Hospital Bern, Bern, Switzerland \\ ${ }^{5}$ Institute of Primary Health Care (BIHAM), University of Bern, Bern, Switzerland \\ ${ }^{6}$ Cardiovascular Center, Department of Cardiology, University Hospital Geneva, Geneva, Switzerland \\ ${ }^{7}$ Royal Brompton \& Harefield Hospital Trust, Imperial College London, London, UK
}

Correspondence should be addressed to Slayman Obeid; Slayman.Obeid@usz.ch and Thomas F. Lüscher; cardio@tomluescher.ch

Received 1 May 2018; Accepted 12 August 2018; Published 25 September 2018

Academic Editor: Stephan von Haehling

Copyright $\odot 2018$ Slayman Obeid et al. This is an open access article distributed under the Creative Commons Attribution License, which permits unrestricted use, distribution, and reproduction in any medium, provided the original work is properly cited.

\begin{abstract}
Aims. To assess the incremental prognostic value of SYNTAX score II (SxSII) as compared to anatomical SYNTAX Score (SxS) and GRACE risk score in patients with acute coronary syndromes who underwent percutaneous coronary intervention. Methods and results. SxSII and SxS were determined in 734 ACS patients. Patients were enrolled in the prospective Special Program University Medicine ACS and the COMFORTABLE AMI cohorts and later on stratified according to tertiles of SxSII (SxSII Low $\leq 21.5$ ( $n=245)$, SxSII $_{\text {Mid }} 21.5-30.6(n=245)$, and SxSII ${ }_{\text {High }} \geq 30.6(n=244)$. The primary endpoint of adjudicated all-cause mortality and secondary endpoints of MACE (cardiac death, repeat revascularization, and myocardial infarction) and MACCE (all-cause mortality, cerebrovascular events, MI, and repeat revascularization) were determined at 1-year follow-up. SxSII provided incremental predictive information for risk stratification when compared to SxS and GRACE risk score (AUC 0.804, 95\% CI 0.77-0.84, $p<0.001$ versus 0.67 , $95 \%$ CI $0.63-0.72, p=0.007$ versus $0.69,95 \%$ CI $0.6-0.8, p=0.002$ ), respectively. In a multivariable Cox regression analysis, we found that unlike SxS (adjusted HR 1.013, 95\% CI $(0.96-1.07), p=0.654)$, SxSII was significantly associated with all-cause mortality $(\mathrm{HR}=1.095,95 \% \mathrm{CI}(1.06-1.11), p<0.001)$. This was also true for the prediction of both secondary outcomes MACE $(n=60)$ and MACCE $(n=70)$ with an adjusted HR $=1.055,95 \%$ CI $(1.03-1.08), p<0.001$, and HR=1.065, 95\% CI $(1.04-1.09), p<0.001$. Conclusion. In patients with ACS who underwent PCI, SxSII is an independent predictor of mortality during 1-year follow-up. SxSII shows superiority in discriminating risk compared to conventional SxS and GRACE for all-cause mortality.
\end{abstract}

\section{Introduction}

The anatomical SYNTAX (Synergy between percutaneous coronary intervention with taxus and cardiac surgery) score $(\mathrm{SxS})$ is an angiographic scoring system for assessing the complexity of coronary artery disease (CAD) [1] advocated for decision making in the latest ESC/EACTS guidelines on myocardial revascularization [2]. Originally, the SxS was introduced to predict clinical outcomes in stable patients with 3-vessel and/or left main disease undergoing percutaneous 
coronary intervention (PCI) or coronary artery bypass grafting (CABG), respectively, based on data from the SYNTAX trial $[3,4]$. Later on, the $\mathrm{SxS}$ was applied to a variety of patient populations with diverse clinical presentations including those with acute coronary syndromes (ACS) undergoing primary PCI $[5,6]$.

However, subanalyses of the SYNTAX trial and results from different studies have implied that the purely anatomybased risk stratification of the SxS score made it prone to misclassification of patient's true risk, particularly for allcause mortality and cardiac death in patients with stable CAD or ACS treated by PCI $[7,8]$. The addition of clinical variables was a promising step in improving risk stratification by reclassifying patients into more accurate risk categories. Therefore, in order to account for the variability of clinical parameters affecting long-term outcomes and hence better classification of patients' risk, the SYNTAX score II (SxSII) was developed by complementing SxS with 7 prognostic variables including age, creatinine clearance, left ventricular ejection fraction (LVEF), presence of unprotected left main coronary artery (ULMCA) disease, peripheral vascular disease (PVD), female gender, and chronic obstructive pulmonary disease (COPD) [9]. So far, the SxSII was validated in patients with left main and multivessel disease showing more accurate patient stratification than SxS $[10,11,12]$.

The aim of the present study was to assess the predictive performance of SxSII in patients presenting with ACS undergoing PCI and to compare it to the previously validated SxS and the commonly used Global Registry of Acute Coronary Events (GRACE) risk score [13].

\section{Methods}

2.1. Study Population. The prospective multicenter Special Program University Medicine-Acute Coronary Syndromes and Inflammation (SPUM-ACS, ClinicalTrials.gov number, NCT01000701) enrolled consecutive patients who were referred for coronary angiography with a diagnosis of ACS to one of the participating Swiss university hospitals (Zurich, Bern, Lausanne, and Geneva) between December 2009 and October 2012 [14, 15]. Inclusion criteria comprised patients of both genders, aged $\geq 18$ years, presenting within 5 days (preferably within 72 hours) after pain onset with a main diagnosis of STEMI, NSTEMI, or unstable angina. Enrolled patients had symptoms compatible with angina pectoris (chest pain and dyspnea) and fulfilled at least one of the following criteria: (a) ECG changes, such as persistent STsegment elevation or depression, T-inversion or dynamic ECG changes, or new left bundle branch block (LBBB); (b) evidence of positive (predominantly conventional) troponin by local laboratory reference values (with a rise and/or fall in serial troponin levels); and (c) known coronary artery disease specified by its status after MI, coronary artery bypass graft $(\mathrm{CABG})$, or PCI or newly documented $\geq 50 \%$ stenosis of an epicardial coronary artery during the initial catheterization. Exclusion criteria for the SxSII study comprised prior $\mathrm{CABG}$, referral to either $\mathrm{CABG}$ or medical management after completion of the coronary angiogram, severe physical disability, and inability to comprehend study or less than 1 year of life expectancy for noncardiac reasons. Within this consortium, a centralized electronic database was implemented providing comprehensive information on all patients comprising both clinical and coronary anatomy parameters. A telephone follow-up was performed at 30 days and at 1 year, a clinical visit. Adverse events occurring within 365 days after the index ACS event were adjudicated by an independent adjudication committee consisting of 3 experienced cardiologists (Lukas Kappenberger, MD, Lausanne; Tiziano Moccetti, MD Lugano; and Mathias E. Pfisterer, MD, Basel). An additional 3-year follow-up to assess all-cause mortality was ascertained by telephone for the SxSII study, and only patients recruited in Bern and Zurich were analyzed in the SxSII study. The study was approved by the local ethical committees, and all patients gave written informed consent in compliance with the Declaration of Helsinki.

The COMFORTABLE AMI trial included patients aged 18 years or older who had a history of chest pain of more than a 10 min duration and associated ST-segment elevation of $>1 \mathrm{~mm}$ in $\geq 2$ contiguous leads, new left bundle branch block, or true posterior MI, who underwent primary percutaneous coronary intervention (PCI) within $24 \mathrm{~h}$ of symptom onset. In addition, there was angiographic presence of at least one acute infarct-related artery (IRA) with one or multiple coronary artery lesions in a native coronary artery with a diameter between 2.25 and $4.0 \mathrm{~mm}$, which could be treated with one or multiple stents. Exclusion criteria included the use of vitamin $\mathrm{K}$ antagonists, mechanical complications of myocardial infarction, acute myocardial infarction secondary to stent thrombosis (ST), planned surgery within 6 months of PCI unless dual antiplatelet therapy could be maintained throughout the perisurgical period, and noncardiac comorbid conditions with life expectancy $<1$ year. Further study details are described in detail elsewhere [16].

Angiography was digitally recorded and analyzed in a central core laboratory. The MI SxS score was assessed by experienced analysts using the web-based program http: //www.syntaxscore.com as previously described. Angiographic documentation of patients included in the COMFORTABLE AMI trial was scored as described previously.

2.2. ClinicalOutcomes. All-cause mortality included cardiac, vascular, and noncardiovascular causes of death. Cerebrovascular events comprised stroke (any, ischemic, hemorrhagic, and unclear etiology) or transient ischemic attack (TIA); repeat revascularization included any repeat coronary revascularization (target and nontarget vessel). Clinically indicated repeat revascularization included any clinically driven repeat coronary revascularization (target and nontarget vessel) [17]. Myocardial infarction was defined based on the universal definition including periprocedural $\mathrm{MI}$ in patients with UA [18].

The primary endpoint of our study was adjudicated all-cause mortality at 1-year follow-up. The secondary 
endpoints were adjudicated major adverse cardiovascular events (MACE) defined as the composite of cardiac death, clinically indicated revascularization, or MI at 1 year and adjudicated major adverse cardiovascular and cerebrovascular events (MACCE) defined as the composite of all-cause mortality, cerebrovascular events, any repeat revascularization, or myocardial infarction (MI) at 1-year, respectively.

2.3. Anatomical SYNTAX Score, SYNTAX Score II, and GRACE Risk Score Calculation. Experienced cardiologists blinded to clinical outcomes assessed the SYNTAX score for each angiogram. The interobserver and intraobserver variabilities of the SxS scoring team were previously reported as moderate (kappa statistic 0.56) and substantial (kappa statistic 0.70 , respectively). The intraclass correlation coefficient for calculated SXS in SPUM cohort for absolute agreement was $0.886(p<0.001), 95 \%$ CI $(0.835,0.919)$. Before accessing any lesions, all those with $\geq 50 \%$ diameter stenosis in vessels $\geq 1.5 \mathrm{~mm}$ in diameter were scored using the SxS algorithm [1]. The SxSII was then calculated using the PCI SYNTAX score II (http://www.syntaxscore.com) calculator based on the previously published nomogram [9], with scores assigned for the presence and magnitude of each predictor specific for PCI population $[9,11]$. The GRACE risk score to calculate long-term mortality comprised age, heart rate, systolic blood pressure, initial serum creatinine, history of congestive heart failure, history of myocardial infarction, elevated cardiac markers (conventional troponins as per local laboratories), ST-segment depression, and no in-hospital PCI [13]. The long-term GRACE risk score was calculated using a program written in STATA, and we used the standard scoring of GRACE as mentioned in the reference publications. (http://www. wikidoc.org/index.php/The_GRACE_risk_score). It was retrospectively calculated for all patients included in our study and was compared to SxSII and $\mathrm{SxS}$ as a continuous variable by ROC curve analysis and multivariable Cox regression model.

2.4. Statistical Analysis. Continuous variables are expressed as mean $\pm \mathrm{SD}$ or medians with interquartile ranges (IQR) and were compared using one-way ANOVA, Student's $t$ test, Kruskal-Wallis test, or Mann-Whitney test as appropriate. Categorical data are presented as frequency (percentages) and were compared using the Fisher exact or the chi-square test. Cumulative incidences were calculated using Kaplan-Meier curves.

For our analysis, we stratified patients according to tertiles of SxSII [9] $(\leq 21.5,21.5-30.6, \geq 30.6)$ and SXS $(\leq 12$, $12-22, \geq 22)$. The score ranges are referred to as $\operatorname{SxSII}_{\text {Low }}$, SxSII ${ }_{\mathrm{Mid}}$, and $\mathrm{SxSII}_{\mathrm{High}}$ and $\mathrm{SxS}_{\text {Low }}, \mathrm{SxS}_{\mathrm{Mid}}$, and $\mathrm{SxS}_{\mathrm{High}}$, respectively. We constructed multivariable Cox proportional hazard models including variables that had a significant association with a $p$ value of $<0.05$ in univariable analysis. We further added SxS and SxSII separately into the model due to their collinearity. Other variables showed no multicollinearity that exceeding the acceptable threshold of
VIF $\geq 3$ or tolerance $\leq 0.2$. From the full model, we selected variables to minimize Akaike's information criterion by backward stepwise methods. Calibration was then determined by the Hosmer-Lemeshow goodness-of-fit test. To visualize the effect of SxSII on clinical outcomes and confirm its linearity, we constructed an alternative model using penalized splines. Analyses were performed with SPSS version 21.0 software (SPSS Inc., Chicago, Ill).

ROC curves were constructed to assess the ability of the SxSII, SxS, and GRACE risk score to predict events at 1year follow-up. Patients who were lost to follow-up at 1 year were excluded from the analysis. Areas under curves were compared using the DeLong method [19] provided by MedCalc for Windows, version 14.10.2 (MedCalc Software, Acacialaan, Belgium). Category-free net reclassification improvement (NRI) and integrated discrimination improvement (IDI) were both calculated using the "survIDINRI," R package (R-version 3.3.2), through comparing proportional hazards models, whereas category-based NRI was done using MATLAB version R2015b, all as described by Pencina et al. [20]. A probability value of $<0.05$ was considered significant, and all tests were two-tailed.

\section{Results}

3.1. Baseline and Angiographic Characteristics for Tertiles of SYNTAX Score II. SxS and SxSII could be calculated for all 734 patients with a complete one-year follow-up. The mean anatomical SYNTAX score was $17.56 \pm 9.3$ with a median of 16 with an interquartile range (IQR) of 13 . Patients were categorized into $\mathrm{SxS}$ tertiles $\left(\mathrm{SxS}_{\mathrm{Low}}, n=251, \mathrm{SxS}_{\mathrm{Mid}}\right.$, $n=244$, and $\left.\mathrm{SxS}_{\mathrm{High}}, n=239\right)$. The mean SYNTAX score II was $27.7 \pm 10.3$ with a median of 25.4. The number of patients stratified according to tertiles of SxSII ${ }_{\text {Low }}, S_{x S I I}$ Mid, and $\mathrm{SxSII}_{\mathrm{High}}$, was 245,245 , and 244 , respectively. The tertiles of SxSII and its individual components including the anatomical SYNTAX score are listed in Table 1 as they are compared to tertiles of the conventional SxS.

Compared with patients in the lower tertiles, patients in SxSII High had a higher rate of adverse cardiovascular history and risk factors and fasting glucose, along with hemodynamic instability on admission. The baseline characteristics and risk factors of patients according to SxSII tertiles are listed in Table 2. Angiographic characteristics showed significant differences with a higher rate of MVD with left anterior descending artery (LAD) involvement $(74.2 \%)$ in the $\mathrm{SxSII}_{\mathrm{High}}$ tertile compared with patients in the lower tertiles (Table 3).

3.2. Clinical Outcomes Stratified by Tertiles of Anatomical SYNTAX and SYNTAX Score II. In order for better assessment of the impact SxSII has on mortality, we expressed this relationship in terms of penalized splines curves (hazard ratio-based curves) obtained through a Cox proportional hazard regression model (Supplementary Figure 1). As the relationship was curve-linear, our choice of breaking the score into tertiles was justified. 
TABLE 1: Baseline characteristics and risk factors.

\begin{tabular}{|c|c|c|c|c|}
\hline Variables & $\begin{array}{c}\text { SxSII }_{\text {Low }} \leq 21.5 \\
N=245 \\
\end{array}$ & $\begin{array}{c}\text { SxSII }_{\mathrm{Mid}} 21.5-30.6 \\
\quad N=245\end{array}$ & $\begin{array}{c}\mathrm{SxSII}_{\mathrm{High}} \geq 30.6 \\
N=244\end{array}$ & $p$ value \\
\hline Age* $^{*}$ & $52.3 \pm 7.4$ & $60.5 \pm 9.5$ & $71.7 \pm 10.2$ & $\mathrm{~N} / \mathrm{A}$ \\
\hline Gender (male) ${ }^{*}$ & $239(97.6)$ & $205(83.7)$ & $146(59.8)$ & N/A \\
\hline ACS & & & & 0.001 \\
\hline STEMI & $167(68.2)$ & $181(73.9)$ & $189(77.5)$ & \\
\hline NSTE-ACS & $65(26.5)$ & $63(25.7 .4)$ & $53(21.7)$ & \\
\hline Unstable angina & $13(5.3)$ & $1(0.4)$ & $2(0.8)$ & \\
\hline Hypertension & $100(43.1)$ & $122(51.7)$ & $155(68)$ & $<0.001$ \\
\hline History of dyslipidemia & $143(61.9)$ & $140(59.6)$ & $138(60.8)$ & 0.878 \\
\hline Diabetes mellitus & $28(12.1)$ & $41(17.4)$ & $39(17.1)$ & 0.207 \\
\hline History of smoking & $207(84.5)$ & $188(76.7)$ & $142(59.2)$ & $<0.001$ \\
\hline History of CAD & $72(31.9)$ & $57(24.2)$ & $38(17.5)$ & 0.002 \\
\hline Prior MI & $11(4.7)$ & $20(8.5)$ & $25(11)$ & 0.049 \\
\hline Prior PCI & $19(8.2)$ & $25(10.5)$ & $28(12.3)$ & 0.347 \\
\hline History of PVD* & $0(0)$ & $1(0.4)$ & $23(9.4)$ & N/A \\
\hline History of COPD* & $3(1.2)$ & $6(2.4)$ & $15(6.1)$ & $\mathrm{N} / \mathrm{A}$ \\
\hline Killip score $\geq 2$ & $21(8.5)$ & $31(12.7)$ & $46(18.9)$ & 0.003 \\
\hline Prehospital resuscitation & $16(6.5)$ & $8(3.3)$ & $10(4.1)$ & 0.226 \\
\hline Vasopressors & $5(2)$ & $4(1.6)$ & $15(6.1)$ & 0.007 \\
\hline IABP & $3(1.2)$ & $9(3.7)$ & $33(13.5)$ & $<0.001$ \\
\hline BMI $\left(\mathrm{kg} / \mathrm{m}^{2}\right)$ & $27.7 \pm 3.9$ & $27.5 \pm 4.2$ & $25.6 \pm 3.6$ & $<0.001$ \\
\hline HsTnT (ug/l) & $0.14(0.04 / 0.51)$ & $0.18(0.04 / 0.67)$ & $0.24(0.08 / 0.73)$ & 0.008 \\
\hline CK-MB (U/l) & $24.3(11 / 57)$ & $28.5(12 / 68)$ & $34(14 / 66)$ & 0.104 \\
\hline NT-proBNP (ng/l) & $137(47 / 311)$ & $256(95 / 701)$ & $586(195 / 1960)$ & $<0.001$ \\
\hline eGFR $\left(\mathrm{ml} / \mathrm{min} / 1.73 \mathrm{~m}^{2}\right)^{*}$ & $127.8 \pm 30.8$ & $106.9 \pm 33.6$ & $69.5 \pm 29.4$ & N/A \\
\hline Fasting glucose $(\mathrm{mmol} / \mathrm{l})$ & $6.8 \pm 2.2$ & $7.4 \pm 2.9$ & $7.8 \pm 3.4$ & 0.001 \\
\hline $\operatorname{LVEF}(\%)^{*}$ & $55.7 \pm 7.4$ & $50.7 \pm 10.2$ & $45.9 \pm 12.4$ & N/A \\
\hline
\end{tabular}

Depicted are counts, $n$ incidence (\%) or mean \pm SD or median (25/75) percentile. ${ }^{*}$ Variables are included in the score; $p$ value reported as N/A. CAD, coronary artery disease; MI, myocardial infarction; PCI, percutaneous coronary intervention; PVD, peripheral vascular disease; COPD, chronic obstructive pulmonary disease; LVEF, left ventricular ejection fraction.

TABLE 2: Components of SYNTAX score II.

\begin{tabular}{|c|c|c|c|c|}
\hline Variables & $\begin{array}{c}\text { SxSII }_{\text {Low }} \leq 21.5 \\
N=245\end{array}$ & $\begin{array}{c}\mathrm{SxSII}_{\mathrm{Mid}} 23-32 \\
N=245\end{array}$ & $\begin{array}{c}\mathrm{SxSII}_{\mathrm{High}} \geq 30.6 \\
N=244\end{array}$ & $p$ value \\
\hline Age & $52.3 \pm 7.4$ & $60.5 \pm 9.5$ & $71.7 \pm 10.2$ & $<0.001$ \\
\hline Gender (male) & $239(97.6)$ & $205(83.7)$ & $146(59.8)$ & $<0.001$ \\
\hline PVD & $0(0)$ & $1(0.4)$ & $23(9.4)$ & $<0.001$ \\
\hline COPD & $3(1.2)$ & $6(2.4)$ & $15(6.1)$ & 0.004 \\
\hline $\mathrm{eGFR}\left(\mathrm{ml} / \mathrm{min} / 1.73 \mathrm{~m}^{2}\right)$ & $127.8 \pm 30.8$ & $106.9 \pm 33.6$ & $69.5 \pm 29.4$ & $<0.001$ \\
\hline $\operatorname{LVEF}(\%)$ & $55.7 \pm 7.4$ & $50.7 \pm 10.2$ & $45.9 \pm 12.4$ & $<0.001$ \\
\hline LM & $2(0.8)$ & $2(0.8)$ & $10(4.1)$ & 0.009 \\
\hline Anatomical SYNTAX score $(\mathrm{SxS})$ & $12.6 \pm 6.9$ & $17.6 \pm 7.8$ & $22.4 \pm 10.2$ & $<0.001$ \\
\hline Variables & $\begin{array}{c}\mathrm{SxS}_{\text {Low }} \leq 12 \\
N=251\end{array}$ & $\begin{array}{c}\mathrm{SxS}_{\mathrm{Mid}} 12-22 \\
N=244\end{array}$ & $\begin{array}{c}S_{x S_{\text {High }} \geq 22} \\
N=239\end{array}$ & $p$ value \\
\hline Anatomical SYNTAX score (SxS) & $7.9 \pm 2.8$ & $16.9 \pm 2.91$ & $28.5 \pm 6.4$ & $<0.001$ \\
\hline
\end{tabular}

PVD, peripheral vascular disease; COPD, chronic obstructive pulmonary disease; LVEF, left ventricular ejection fraction; GFR, glomerular filtration rate; LM, left main disease.

Patients within the SxSII $\mathrm{High}_{\text {}}$ tertile had a significantly higher incidence of all-cause mortality, MACE, and MACCE compared with patients in lower tertiles (Table 4). There was also a higher rate of clinically driven revascularization, in $\mathrm{SxSII}_{\text {High }}(8.6 \% p=0.002)$ with a trend for excess cerebrovascular events, $p=0.134$. One-year outcomes across the tertiles of SxS are reported in Supplementary Table 1.

Furthermore, Kaplan-Meier curves were plotted to asses all studied outcomes across tertiles of both SxS and SxSII as shown in Figure 1. All-cause mortality (9.4\% versus $1.2 \%$ versus $0.8 \%$ ), MACCE (17.6\% versus $8.6 \%$ versus $2.4 \%$ ), and MACE (14.3\% versus $8.2 \%$ versus $2 \%$ ) occurred at a significantly higher rate among patients in $\mathrm{SxSII}_{\mathrm{High}}$ compared to $\mathrm{SxSII}_{\mathrm{Mid}}$ and $\mathrm{SxSII}_{\mathrm{Low}}$, respectively ( $p$ (log rank) $<0.001)$. Conversely, the anatomical SYNTAX score does not provide consistent risk stratification, specifically when addressing the primary endpoint of all-cause mortality at 1 year. 
TABle 3: Angiographic characteristics and medications.

\begin{tabular}{|c|c|c|c|c|}
\hline Variables & $\begin{array}{c}\mathrm{SxSII}_{\text {Low }}<23 \\
N=176\end{array}$ & $\begin{array}{c}\mathrm{SxSII}_{\mathrm{Mid}} 23-32 \\
\quad N=164 \\
\end{array}$ & $\begin{array}{c}\text { SxSII }_{\text {High }} \geq 23 \\
N=160 \\
\end{array}$ & $p$ value \\
\hline \multicolumn{5}{|l|}{ Vessel involvement } \\
\hline $\mathrm{LM}^{*}$ & $2(0.8)$ & $2(0.8)$ & $10(4.1)$ & N/A \\
\hline LAD & $121(49.4)$ & $157(64.1)$ & $181(74.2)$ & $<0.001$ \\
\hline $\mathrm{LCx}$ & $72(29.4)$ & $83(34)$ & $84(34.4)$ & 0.419 \\
\hline $\mathrm{RCA}$ & $125(51)$ & $126(51.6)$ & $134(54.9)$ & 0.654 \\
\hline$M V D \#$ of vessels & $86(35.1)$ & $106(43.3)$ & $130(53.3)$ & \multirow[t]{3}{*}{$<0.001$} \\
\hline 2 vessels & $72(29.4)$ & $71(29)$ & $83(34)$ & \\
\hline 3 vessels & $14(5.7)$ & $35(14.3)$ & $47(19.3)$ & \\
\hline ACC/AHA lesion classification & 0.413 & & & \multirow[t]{5}{*}{0.413} \\
\hline Type A lesion & $15(9.6)$ & $18(11.7)$ & $10(7.0)$ & \\
\hline Type B1 lesion & $73(46.8)$ & $56(36.4)$ & $60(42.3)$ & \\
\hline Type B2 lesion & $41(26.3)$ & $45(29.2)$ & $37(26.1)$ & \\
\hline Type C lesion & $27(17.3)$ & $35(22.7)$ & $35(24.6)$ & \\
\hline Restenotic lesion & $7(2.9)$ & $6(2.4)$ & $5(2.1)$ & 0.876 \\
\hline Bifurcation lesion & $20(8.3)$ & $28(11.5)$ & $27(11.2)$ & 0.441 \\
\hline Thrombus Procedural details & $132(54.3)$ & $120(49)$ & $107(44.2)$ & \multirow{7}{*}{0.083} \\
\hline Length of 1 st stent & $25.4 \pm 5$ & $20.2 \pm 5$ & $25.4 \pm 5$ & \\
\hline Diameter of 1 st stent & $3.03 \pm 0.68$ & $2.9 \pm 0.41$ & $2.81 \pm 0.22$ & \\
\hline Length of 2 nd stent & $19.85 \pm 6.83$ & $23.6 \pm 6.12$ & $18.2 \pm 5.6$ & \\
\hline Diameter of 2 nd stent & $3.1 \pm 0.28$ & $3 \pm 0.68$ & $2.6 \pm 0.13$ & \\
\hline Length of 3 rd stent & $15.9 \pm 7.02$ & $12.4 \pm 2.8$ & $16.2 \pm 9$ & \\
\hline Diameter of $3 \mathrm{rd}$ stent & $3.17 \pm 0.31$ & $2.95 \pm 0.77$ & $2.65 \pm 0.48$ & \\
\hline TIMI flow & & & & 0.207 \\
\hline TIMI 0 & $107(44.4)$ & $134(54.9)$ & $128(53.1)$ & \\
\hline TIMI I & $20(8.3)$ & $15(6.1)$ & $10(4.1)$ & \\
\hline TIMI II & $38(15.8)$ & $33(13.5)$ & $36(14.9)$ & \\
\hline TIMI III & $76(31.5)$ & $62(25.4)$ & $67(27.8)$ & \\
\hline \multicolumn{5}{|l|}{ Medications on admission } \\
\hline ASA & $213(86.9)$ & $205(83.7)$ & $159(65.7)$ & $<0.001$ \\
\hline Clopidogrel & $6(2.5)$ & $7(2.9)$ & $14(5.8)$ & 0.125 \\
\hline Prasugrel/ticagrelor & $1(0.6)$ & $0(0)$ & $0(0)$ & 1 \\
\hline Statin & $34(14)$ & $48(19.6)$ & $63(26.1)$ & 0.004 \\
\hline Beta blocker & $31(12.7)$ & $43(17.6)$ & $68(28.3)$ & $<0.001$ \\
\hline \multicolumn{5}{|l|}{ Procedural antiplatelet $T x$} \\
\hline Clopidogrel & $119(69.2)$ & $120(73.2)$ & $117(73.6)$ & 0.451 \\
\hline Prasugrel & $63(35.8)$ & $46(28.0)$ & $26(16.4)$ & $<0.001$ \\
\hline
\end{tabular}

Depicted are counts, $n$ incidence (\%) or mean \pm SD. ${ }^{*}$ variables are included in the score; $p$ value reported as N/A. LAD, left anterior descending artery; RCA, right coronary artery; LCX, circumflex artery; TIMI, thrombolysis in myocardial infarction; AHA, American Heart Association.

TABLE 4: Clinical outcomes at 1 year stratified by tertiles of SYNTAX score II.

\begin{tabular}{|c|c|c|c|c|c|}
\hline Outcomes at one year & $\begin{array}{c}\text { SxSII }_{\text {Low }} \leq 21.5 \\
N=245\end{array}$ & $\begin{array}{c}\mathrm{SxSII}_{\mathrm{Mid}} 21.5-30.6 \\
N=245\end{array}$ & $\begin{array}{c}\text { SxSII }_{\text {High }} \geq 30.6 \\
N=244\end{array}$ & $p$ value & $N=734$ \\
\hline All-cause mortality & $2(0.8)$ & $3(1.2)$ & $23(9.4)$ & $<0.001$ & $28(3.8)$ \\
\hline Cardiovascular death & $1(0.4)$ & $3(1.2)$ & $17(7.0)$ & $<0.001$ & $21(2.8)$ \\
\hline Noncardiovascular death & $1(0.4)$ & $0(0.0)$ & $6(2.4)$ & 0.007 & $7(0.9)$ \\
\hline Cerebrovascular event & $0(0.0)$ & $1(0.4)$ & $3(1.2)$ & 0.134 & $4(0.5)$ \\
\hline Myocardial infarction & $1(0.4)$ & $9(3.7)$ & $10(4.1)$ & 0.011 & $20(2.7)$ \\
\hline Clinically driven revascularization & $4(1.6)$ & $16(6.5)$ & $21(8.6)$ & 0.002 & $41(5.6)$ \\
\hline Target vessel revascularization & $2(1.7)$ & $13(5.3)$ & $13(5.3)$ & 0.011 & $28(3.8)$ \\
\hline Any revascularization & $4(1.6)$ & $18(7.3)$ & $21(8.6)$ & 0.002 & $43(5.8)$ \\
\hline Restenotic lesion revascularisation & $3(1.7 \%)$ & $2(1.2 \%)$ & $9(1.8 \%)$ & 0.03 & $14(1.9)$ \\
\hline Any stent thrombosis & $2(1.1 \%)$ & $0(0 \%)$ & $3(1.9 \%)$ & 0.59 & $5(0.6)$ \\
\hline Early thrombosis & $1(0.6 \%)$ & $0(0 \%)$ & $2(1.3 \%)$ & 0.50 & $3(0.4)$ \\
\hline Late thrombosis & $1(0.6 \%)$ & $0(0 \%)$ & $1(0.6 \%)$ & 0.77 & $2(0.2)$ \\
\hline MACE & $5(2.0)$ & $20(8.2)$ & $35(14.3)$ & $<0.001$ & $60(7.4)$ \\
\hline MACCE & $6(2.4)$ & $21(8.6)$ & $43(17.6)$ & $<0.001$ & $70(9.5)$ \\
\hline
\end{tabular}

MACE, major adverse cardiac events; MACCE, major adverse cardiac and cerebrovascular events. 


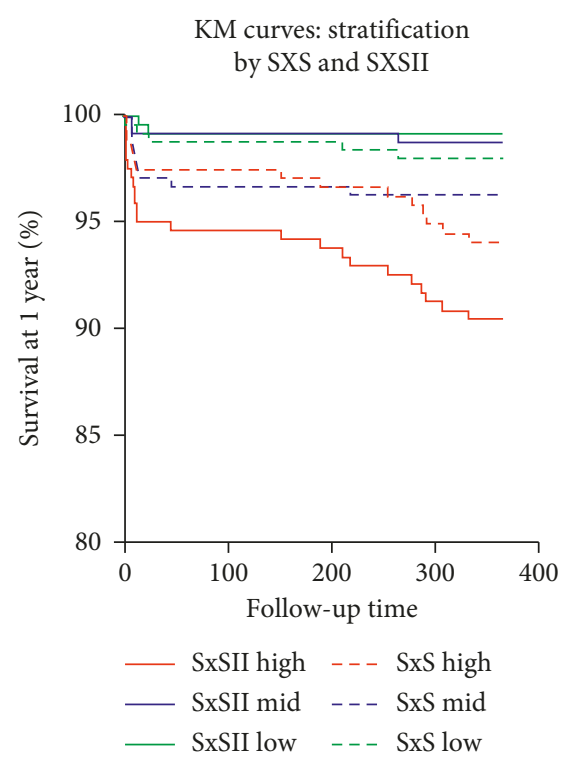

(a)

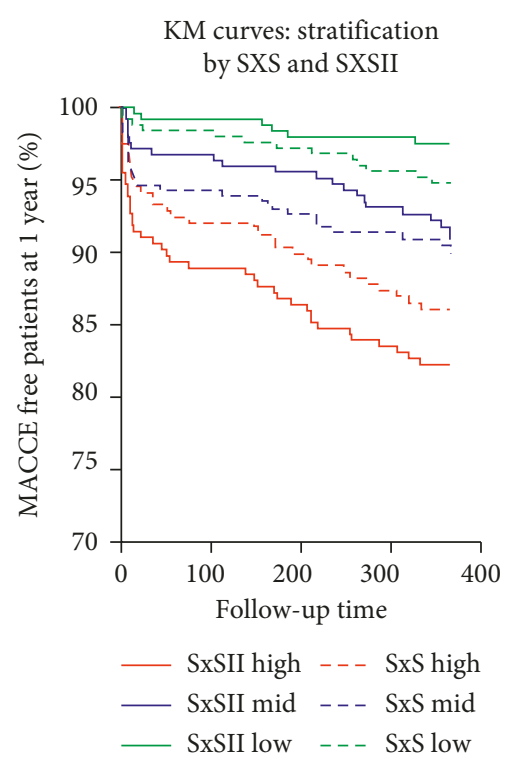

(b)

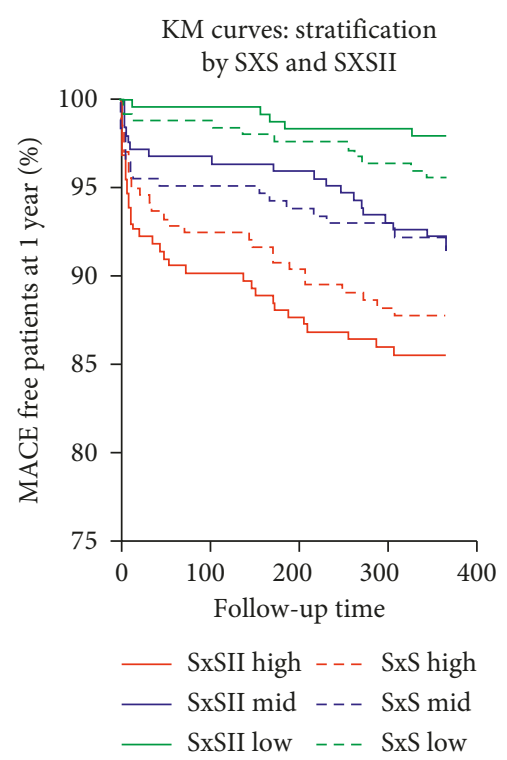

(c)

FIgURE 1: Kaplan-Meier survival curves at 1-year follow-up for freedom from all-cause mortality, MACCE, and MACE.

3.3. A Comparison between SxSII, SxS, and GRACE Score. ROC curves showed an improved area under the curve (AUC) when comparing SxSII with SxS regarding all-cause mortality at 1 year $(0.803(0.773-0.831)$ versus 0.658 $(0.622-0.692)$ ) (Figure 2(a)). This improvement was statistically significant at an AUC difference of 0.145 (95\% CI $0.049-0.246, p=0.0045)$. Compared with the GRACE risk score (calculated in 500 patients), SxSII showed a persistently higher prognostic accuracy for all-cause mortality (Figure 2(b)). Conversely, prognostic accuracy for MACE during 1-year follow-up (AUC (0.657 (0.621-0.691) versus $\left.0.684(0.649-0.718), p_{\text {Difference }}=0.475\right)$ was not different between SxS and SxSII (Supplementary Figure 2).

Additionally, we sought to authenticate the benefit in risk assessment, that we have seen so far by SxSII over SXS, through performing a category-free and categorybased net reclassification improvement (NRI) along with integrated discrimination improvement (IDI) for the outcome of all-cause mortality at 1 year. We found a significant categorical net reclassification improvement of $0.344(p=0.004)$ with Z score of 2.714 for all-cause mortality, primarily driven by a significant net gain of $0.321 \quad(p=0.006)$ in patients who had the event and a trend $(p=0.39)$ in patients without the event (Table 5). More importantly, both estimates of category-free net reclassification improvement (NRI) and integrated discrimination improvement (IDI) were significant $(p<0.001)$ at $0.38095 \% \mathrm{CI}(0.191-0.554)$ and $0.0895 \% \mathrm{CI}$ (0.035-0.278), respectively. The red shaded area and its extent show a substantial added value of the SxSII model over SxS (Figure 3).

3.4. Results from Multivariable Analysis. For model construction, variables which were found to correlate significantly with the studied outcomes were plotted (Supplementary Figures 3 and 4 ) as part of hazard assessment in a univariable Cox regression analysis. The anatomical SYNTAX score, age, LVEF, and GFR were predictive of the primary outcome; however, they correlated significantly with SxSII while at the same time showing multicollinearity exceeding the acceptable threshold of $\mathrm{VIF}<3$. Hence, two separate models for SxSII and SxS were constructed, showing the SxSII to be a significant predictor of all-cause mortality (adjusted HR 1.095 95\% CI (1.051.13), $p<0.001$ ) along with the presence of left main disease, fasting glucose, and resuscitation status (Table 6). On the other hand, the anatomical SYNTAX score got short of significance for the prediction of our primary outcome (adjusted HR 1.013 95\% CI (0.956-1.073), $p=0.656)$. The complete list of predictors in multivariable analysis for the outcomes of MACE and MACCE is shown in Supplementary Table 2.

Additional analysis regarding the impact of categorizing patients within tertiles of SxSII showed that a classification into $\mathrm{SxSII}_{\mathrm{High}}$ independently predicted all-cause mortality (HR 12.48, 95\% CI (2.61-59.01], $p=0.002$ ), MACCE (HR 7.310, 95\% CI (2.90-18.3), $p<0.001$ ), and MACE (HR 6.64, $95 \%$ CI $(2.42-18.18), p<0.001)$ as compared to the reference category SxSII $_{\text {Low }}$.

Further evaluation of the value of the SxSII over the established GRACE risk score, which can be assessed without information on coronary anatomy, was conducted through an additional multivariable Cox regression model to predict 1-year all-cause mortality. The results showed that SxSII unlike GRACE score remained to be significantly predictive of mortality (adjusted HR 1.061 95\% CI (1.011.11), $p=0.014$ ) (Supplementary Table 3).

\section{Discussion}

To the best of our knowledge, this is the first study to evaluate and compare the novel SxSII score in patients with 


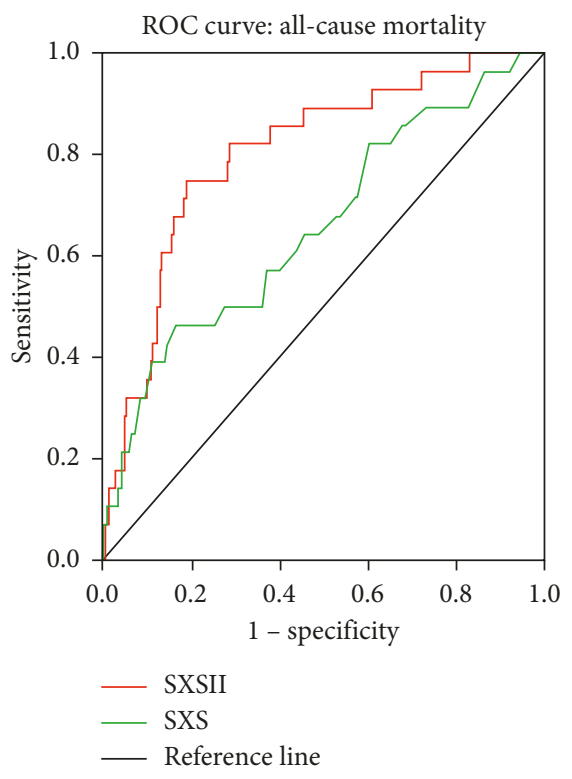

(a)

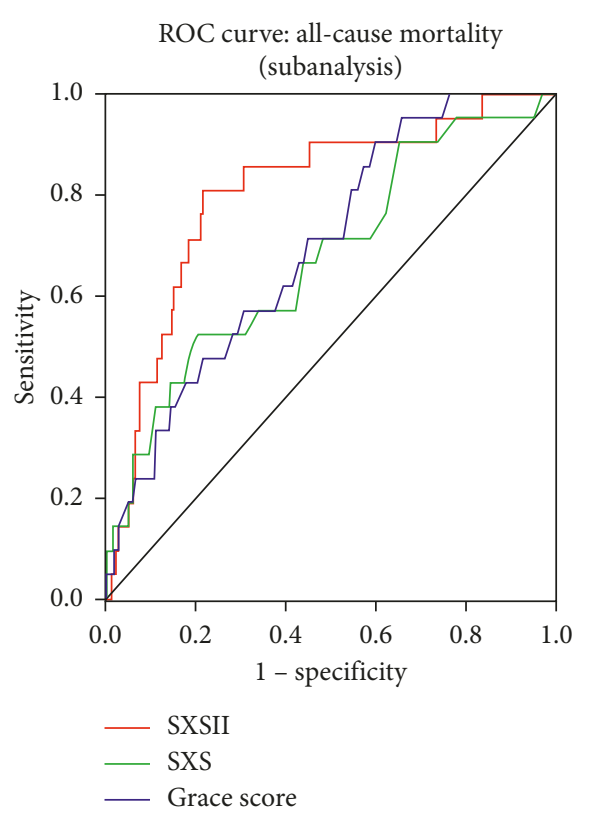

(b)

\begin{tabular}{lll}
\hline AUC SXSII (mortality) & $0.804(0.708-0.899)$ & $p<0.001$ \\
AUC GRACE (mortality) & $0.697(0.594-0.799)$ & $p=0.002$ \\
AUC SXS (mortality) & $0.675(0.552-0.799)$ & $p=0.007$ \\
\hline
\end{tabular}

FIGURE 2: ROC curves. (a) Receiver-operating characteristic (ROC) curves for SYNTAX score II and anatomical SYNTAX in predicting 1year all-cause mortality (entire population of 734 patients). SxSII (red line) significantly improves prediction over both scores. AUC $=$ area under the curve; $\mathrm{CI}=$ confidence intervals. (b) Receiver-operating characteristic (ROC) curves for SYNTAX score II, anatomical SYNTAX, and GRACE risk score in predicting 1-year all-cause mortality (subanalysis of 500 patients).

ACS undergoing PCI with the currently available SxS and GRACE risk scores. The following key findings were obtained:

(i) SxSII independently predicts all-cause mortality, MACE, and MACCE during 1-year follow-up.

(ii) SxSII provides superior discrimination of risk for allcause mortality and MACCE than the conventional SxS and GRACE score, respectively.

In the current era of interventional cardiology, the role of the multidisciplinary HeartTeam in choosing the optimal available means of revascularization is emphasized by current ESC guidelines [2] advocating the use of the anatomical SYNTAX score as a fundamental tool to assist in the decision making for surgical versus percutaneous coronary revascularization in stable coronary artery disease patients with left main or multivessel disease [2]. However, risk assessment and the prediction of long-term outcomes in patients presenting with ACS undergoing PCI is still suboptimal with a wide range of old and newly emerging risk scores [10].

The SxS has been extensively studied for a variety of clinical outcomes in different patient populations including all-comers [21, 22] as well as patients with NSTEMI [23, 24] or STEMI $[25,26]$. The recently developed SxSII has been complemented with clinically significant prognostic variables, known to be independent predictors of mortality at 4 years in patients with stable CAD enrolled in the SYNTAX trial $[9,25,27]$. In that patient population, this was translated into better discrimination of risk for long-term mortality for SxSII when compared to SxS [9]. The main findings from our study support the superiority of SxSII also in patients with ACS undergoing PCI who are at particular risk as it was found to be an independent predictor of allcause mortality during 1-year follow-up. In contrast, the anatomical SYNTAX score was not an independent predictor for this endpoint, in line with previous studies $[22,28,29]$. The discrepant findings between studies evaluating the anatomical SYNTAX score to predict adverse clinical outcome (particularly all-cause mortality) are likely attributable to the heterogeneous patient population analyzed (inclusion or exclusion of patients with STEMI and/or cardiogenic shock). Indeed, the latter patients tend to have a worse outcome which is not predicted by anatomical complexity alone. Furthermore, different cutoffs used in statistical models to calculate SxS may explain the observed differences.

To further substantiate the significant improvement in discrimination or risk for all-cause mortality identified for SxSII, we compared this score to the clinically based, ESCadvocated risk stratification in patients with NSTEMI [2], the GRACE risk score [13]. Despite the fact that patients with cardiogenic shock with high GRACE risk scores were included in our cohort, the SxSII showed greater discrimination of risk for all-cause mortality during 1-year follow- 
TABle 5: Category-based NRI by SxSII.

\begin{tabular}{|c|c|c|c|c|c|}
\hline & \multicolumn{3}{|c|}{ Tertiles of SxSII } & \multirow{2}{*}{$\begin{array}{c}\text { Number o } \\
\text { patients }\end{array}$} \\
\hline & & Low & Mid & High & \\
\hline \multicolumn{6}{|c|}{ Patients with an event } \\
\hline \multirow{3}{*}{ Tertiles of SxS } & Low & 1 & 1 & 3 & $\begin{array}{c}5 \\
17.90 \%\end{array}$ \\
\hline & Mid & 1 & 2 & 6 & $\begin{array}{c}9 \\
32.10 \%\end{array}$ \\
\hline & High & 0 & 0 & 14 & $\begin{array}{c}14 \\
50 \%\end{array}$ \\
\hline \multicolumn{2}{|c|}{ All-cause mortality } & $\begin{array}{c}2 \\
7.10 \%\end{array}$ & $\begin{array}{c}3 \\
10.70 \%\end{array}$ & $\begin{array}{c}23 \\
21.10 \%\end{array}$ & $\begin{array}{c}28 \\
100.00 \%\end{array}$ \\
\hline \multicolumn{6}{|c|}{ Patients without an event } \\
\hline \multirow{3}{*}{ Tertiles of SxS } & Low & 134 & 67 & 45 & $\begin{array}{c}246 \\
34.80 \%\end{array}$ \\
\hline & Mid & 79 & 97 & 59 & $\begin{array}{c}235 \\
33.30 \%\end{array}$ \\
\hline & High & 30 & 78 & 117 & $\begin{array}{c}225 \\
31.90 \%\end{array}$ \\
\hline \multicolumn{2}{|c|}{ All-cause mortality } & $\begin{array}{c}243 \\
34.40 \%\end{array}$ & $\begin{array}{c}242 \\
34.30 \%\end{array}$ & $\begin{array}{c}221 \\
31.10 \%\end{array}$ & $\begin{array}{c}709 \\
100.00 \%\end{array}$ \\
\hline
\end{tabular}

Number of patients with events moving 1 scale up by SxSII $=10$, number of patients with events moving 1 scale down by SxSII $=1$, number of patients without events moving 1 scale down by SxSII $=187$, and number of patients without events moving 1 scale up by $\mathrm{SxSII}=171$. Category-based $\mathrm{NRI}=0.344, z=2.833, p=0.004$.

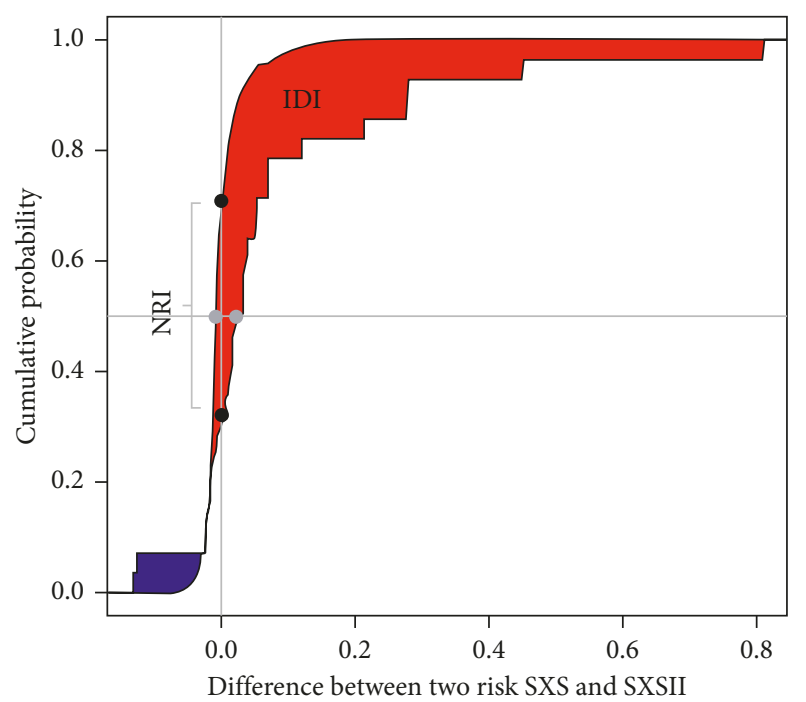

FIGURE 3: Category-free IDI and NRI. The red shaded area shows a significant integrated discrimination improvement (IDI), whereas the difference between the two vertical dots represents the improvement in net reclassification by SXSII over SxS.

up. The GRACE risk score lacks some clinical variables which are important predictors of mortality, such as LVEF, as well as classifiers of complex coronary anatomy [30-33], which may explain the reduced prognostic accuracy to predict all-cause mortality when compared with the SxSII.

In our study, SxSII also showed good prognostic accuracy for the secondary endpoint of MACCE. Conversely, SxSII provided no incremental risk stratification for MACE compared with SxS. This could be explained by the fact that
TABLE 6: Multivariable predictors of all-cause mortality at one year.

\begin{tabular}{|c|c|c|}
\hline \multicolumn{2}{|l|}{ HR $(95 \%$ CI $)$} & \multirow{2}{*}{$\begin{array}{c}p \text { value } \\
\text { All-cause } \\
\text { mortality }\end{array}$} \\
\hline Variables in SxSII model & & \\
\hline SxSII & $1.095(1.05-1.13)$ & $<0.001$ \\
\hline LM disease & $4.825(1.40-16.59)$ & 0.0125 \\
\hline Fasting glucose & $1.081(1.01-1.15)$ & 0.0268 \\
\hline Resuscitation & $11.48(4.53-29.06)$ & $<0.001$ \\
\hline Gender (male) & $0.369(0.126-1.08)$ & 0.0685 \\
\hline H\&L test: $X^{2}: 3.156, d f: 8, p: 0.9$ & & \\
\hline Variables in SXS model & & $\begin{array}{l}\text { All-cause } \\
\text { mortality }\end{array}$ \\
\hline Age & $1.106(1.05-1.16)$ & $<0.0001$ \\
\hline GFR & $1.011(0.99-1.02)$ & 0.14 \\
\hline LVEF & $0.937(0.90-0.97)$ & $<0.001$ \\
\hline LM disease & $3.491(0.93-12.9)$ & 0.06 \\
\hline Resuscitation & $19.7(6.97-55.6)$ & $<0.0001$ \\
\hline Fasting glucose & $1.13(1.05-1.23)$ & 0.0015 \\
\hline SXS & $1.013(0.956-1.073)$ & 0.656 \\
\hline
\end{tabular}

MACE, which were primarily attributable to MI and clinically indicated revascularization in a population with complex coronary anatomy (i.e., a mean SxS of $17.56 \pm 9.3$, 44\% MVD and 63.5\% LAD involvement), could be well predicted by pure anatomical complexity [34-36] as assessed by SxS. Indeed, in our multivariable analysis, multivessel disease was a predictor of MACE and MACCE when assessed with both models of SxSII. However, it fell short of significance for all-cause mortality along with the anatomical which is concordant with previously published studies [6].

Importantly, SxSII enabled reclassification of risk for allcause mortality compared with SxS. This was highly significant for 1-year follow-up with a persistent net gain of approximately $33 \%$. Upon exploring the differences in complexity of the coronary anatomy between tertiles of SxSII and $\mathrm{SxS}$, patients would still classify in the middle category of the original anatomical SxS, although the anatomical SYNTAX score in the $\mathrm{SxSII}_{\mathrm{High}}$ group was higher as com-

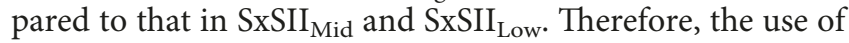
SxSII for risk stratification in patients with ACS undergoing PCI identifies a distinctive group of patients who despite having moderately complex coronary artery disease are still at a significantly higher risk of both cardiovascular and noncardiovascular death during follow-up. These are patients with multiple risk factors and comorbidities who may benefit from intensive secondary prevention, particularly from some of the most recent lipid-lowering drugs [14, 3739] and risk factor modification with closer follow-up intervals and may derive an advantage of full revascularization when multivessel disease is present.

Surprisingly enough, diabetes was not a predictor of clinical outcomes, which could be explained by the fact that end-organ damage secondary to diabetes-as reflected by scores reflecting coronary anatomy and calcification-rather than metabolic parameters better reflect risk in this patient population $[9,13,40]$. Interestingly, abnormal fasting glucose levels during hospitalization for the index event were predictive of all-cause mortality irrespective of the diabetes 
status of the patients. Indeed, in line with previous studies, fasting glucose was also an independent variable capable of predicting all-cause mortality in this population [41-44]. The pathophysiological mechanisms are well described elsewhere [45-47], including but not limited to the effects on the collateral circulation, infarct size, reperfusion, sympathetic activation with elevated catecholamine levels leading to hepatic glucose release and platelet aggregation.

4.1. Study Limitations. One limitation of our study is the fact that due to the low number of patients undergoing CABG or medical therapy in this ACS population, risk by SxSII in the primary PCI group could not be compared to these different treatment modalities. Furthermore, since the SxSII was developed using prognostic variables in a population where STEMI patients were excluded, there may be additional parameters that could further improve risk stratification of adverse clinical outcome.

\section{Conclusion}

In the present study, we demonstrate a clinically relevant superiority of the novel SYNTAX score II when compared to the anatomical SYNTAX and commonly used GRACE risk score, in risk stratification of patients with ACS undergoing PCI. Pending validation in other cohorts, our data suggest that the use of the SYNTAX score II opens a new door for improvement in decision making and management of patients with ACS.

\section{Abbreviations}

$\begin{array}{ll}\text { ACS: } & \text { Acute coronary syndromes } \\ \text { AUC: } & \text { Area under the curve } \\ \text { CABG: } & \text { Coronary artery bypass graft } \\ \text { CI: } & \text { Confidence intervals } \\ \text { ECG: } & \text { Electrocardiogram } \\ \text { ESC: } & \text { European Society of Cardiology } \\ \text { GRACE: } & \text { Global Registry of Acute Coronary Events } \\ \text { LBBB: } & \text { Left bundle branch block } \\ \text { MI: } & \text { Myocardial infarction } \\ \text { NSTEMI: } & \text { Non-ST-elevation myocardial infarction } \\ \text { PCI: } & \text { Percutaneous coronary intervention } \\ \text { ROC: } & \text { Receiver-operating characteristics. }\end{array}$

\section{Data Availability}

The data used to support the findings of this study are available from the corresponding author upon request.

\section{Disclosure}

CTU Bern, which is part of the University of Bern, has a staff policy of not accepting honoraria or consultancy fees. However, CTU Bern is involved in design, conduct, or analysis of clinical studies funded by several companies and nonprofit organizations. Slayman Obeid, Antonio H. Frangieh, and Lorenz Räber contributed equally.

\section{Conflicts of Interest}

LR received speaker fees and research grants to the institution from St. Jude Medical. SW has received research grants to the institution from Abbott, Boston Scientific, Biosensors, Biotronik, the Medicines Company, Medtronic, and St. Jude Medical and honoraria from Abbott, Astra Zeneca, Eli Lilly, Boston Scientific, Biosensors, Biotronik, Medtronic, and Edwards. CMM received research grants to the institution from Eli Lilly, AstraZeneca, Roche, and MSD including speaker or consultant fees. RK received speaker fees from Eli Lilly, Servier, and Bayer Healthcare. TFL received research grants to the institution from AstraZeneca, Bayer Healthcare, Biosensors, Biotronik, Boston Scientific, Eli Lilly, Medtronic, MSD, Merck, Roche, and Servier, including speaker fees by some of them. All other authors have no conflict of interest to declare.

\section{Acknowledgments}

We appreciate the work of the clinical event committee for SPUM ACS: Matthias Pfisterer, MD, University of Basel (chair); Tiziano Moccetti, MD, CardioCentro Lugano; and Lukas Kappenberger, MD, University of Lausanne, Switzerland. We thank the local study nurses (Anika Adam, Maja Müller, Christa Schönenberger, Therese Fahrni, and Saskia Bühlmann), the central data monitors (Katja Heinimann, Daria Bochenek, and Timon Spörri), the electronic data capturing system (2mt GmbH Ulm, Jürgen Nagler-Ihlein, Torsten Illmann), the research coordinator Lambertus J. van Tits, $\mathrm{PhD}$, and Sven Trelle, MD, CTU Bern, and the members of the local catheter teams for their invaluable work. The authors received support from the Swiss National Science Foundation (SPUM 33CM30124112); the Swiss Heart Foundation; the Foundation Leducq; and the Foundation for Cardiovascular ResearchZurich Heart House, Zurich. The SPUM consortium was also supported by Roche Diagnostics, Rotkreuz, Switzerland (providing the kits for the biomarkers), Eli Lilly, Indianapolis (USA), AstraZeneca, and Zug; Medtronic, Münchenbuchsee; Merck Sharpe and Dome (MSD), Lucerne; Sanofi-Aventis, and Vernier; St. Jude Medical, Zurich (all Switzerland).

\section{Supplementary Materials}

Supplementary Figure 1: spline curve representation of different cutoffs of SxSII score and their respective hazard for the outcome of all-cause mortality. Supplementary Figure 2: ROC curves. Supplementary Figures 3 and 4: univariable predictors of studied outcomes. Supplementary Table 1: clinical outcomes at 1 year stratified by tertiles of anatomical SYNTAX score. Supplementary Table 2: multivariable predictors of MACE and MACCE at one year. Supplementary Table 3: GRACE versus SxSII score in prediction of 1-year all-cause mortality. (Supplementary Materials)

\section{References}

[1] G. Sianos, M. A. Morel, A. P. Kappetein et al., "The SYNTAX Score: an angiographic tool grading the complexity of 
coronary artery disease," EuroIntervention: Journal of EuroPCR in Collaboration with the Working Group on Interventional Cardiology of the European Society of Cardiology, vol. 1, no. 2, pp. 219-227, 2005.

[2] Authors/Task Force members, S. Windecker, P. Kolh et al., "ESC/EACTS Guidelines on myocardial revascularization: the task force on myocardial revascularization of the European Society of Cardiology (ESC) and the european association for cardio-thoracic surgery (EACTS) developed with the special contribution of the European association of percutaneous cardiovascular interventions (EAPCI)," European Heart Journal, vol. 35, no. 37, pp. 2541-2619, 2014.

[3] P. W. Serruys, Y. Onuma, S. Garg et al., "Assessment of the SYNTAX score in the Syntax study," EuroIntervention, vol. 5, no. 1, pp. 50-56, 2009.

[4] P. W. Serruys, M. C. Morice, A. P. Kappetein et al., "Percutaneous coronary intervention versus coronary-artery bypass grafting for severe coronary artery disease," New England Journal of Medicine, vol. 360, no. 10, pp. 961-972, 2009.

[5] D. Capodanno, M. E. Di Salvo, G. Cincotta, M. Miano, C. Tamburino, and C. Tamburino, "Usefulness of the SYNTAX score for predicting clinical outcome after percutaneous coronary intervention of unprotected left main coronary artery disease," Circulation Cardiovascular Interventions, vol. 2, no. 8, pp. 302-308, 2009.

[6] A. Caixeta, P. Genereux, T. Palmerini et al., "Prognostic utility of the SYNTAX score in patients with single versus multivessel disease undergoing percutaneous coronary intervention (from the acute catheterization and urgent intervention triage strategy [ACUITY] trial)," American Journal of Cardiology, vol. 163, no. 3, pp. 203-210, 2014.

[7] P. W. Serruys, V. Farooq, P. Vranckx et al., "A global risk approach to identify patients with left main or 3-vessel disease who could safely and efficaciously be treated with percutaneous coronary intervention: the SYNTAX Trial at 3 years," JACC Cardiovascular Interventions, vol. 5, no. 6, pp. 606-617, 2012.

[8] P. C. Keelan, J. M. Johnston, T. Koru-Sengul et al., "Comparison of in-hospital and one- year outcomes in patients with left ventricular ejection fractions <or $=40 \%, 41 \%$ to $49 \%$, and $>$ or $=50 \%$ having percutaneous coronary revascularization," American Journal of Cardiology, vol. 91, no. 10, pp. 1168-1172, 2003.

[9] V. Farooq, D. van Klaveren, E. W. Steyerberg et al., “Anatomical and clinical characteristics to guide decision making between coronary artery bypass surgery and percutaneous coronary intervention for individual patients: development and validation of SYNTAX score II," The Lancet, vol. 381, no. 9867, pp. 639-650, 2013.

[10] V. Farooq, S. J. Head, A. P. Kappetein, and P. W. Serruys, "Widening clinical applications of the SYNTAX Score," Heart, vol. 100, no. 4, pp. 276-287, 2014.

[11] C. M. Campos, D. van Klaveren, J. Iqbal et al., "Predictive performance of SYNTAX score II in patients with left main and multivessel coronary artery disease-analysis of CREDOKyoto registry," Circulation Journal, vol. 78, no. 8, pp. 1942-1949, 2014.

[12] B. Xu, P. Genereux, Y. Yang et al., "Validation and comparison of the long-term prognostic capability of the SYNTAX score-II among 1,528 consecutive patients who underwent left main percutaneous coronary intervention," JACC: Cardiovascular interventions, vol. 7, no. 10, pp. 11281137, 2014.
[13] K. A. Eagle, M. J. Lim, O. H. Dabbous et al., "A validated prediction model for all forms of acute coronary syndrome: estimating the risk of 6-month postdischarge death in an international registry," JAMA, vol. 291, no. 22, pp. 2727-2733, 2004.

[14] B. Gencer, F. Montecucco, D. Nanchen et al., "Prognostic value of PCSK9 levels in patients with acute coronary syndromes," European Heart Journal, vol. 37, no. 6, pp. 546-553, 2016.

[15] R. Klingenberg, D. Heg, L. Raber et al., "Safety profile of prasugrel and clopidogrel in patients with acute coronary syndromes in Switzerland," Heart, vol. 101, no. 1, pp. 854-863, 2015.

[16] M. Magro, L. Raber, D. Heg et al., “The MI SYNTAX score for risk stratification in patients undergoing primary percutaneous coronary intervention for treatment of acute myocardial infarction: a substudy of the COMFORTABLE AMI trial," International Journal of Cardiology, vol. 175, no. 2, pp. 314-322, 2014.

[17] D. E. Cutlip, S. Windecker, R. Mehran et al., "Clinical end points in coronary stent trials: a case for standardized definitions," Circulation, vol. 115, no. 17, pp. 2344-2351, 2007.

[18] K. Thygesen, J. S. Alpert, and H. D. White, "Joint ESC/ACCF/ AHA/WHF expert consensus document: universal definition of myocardial infarction," Journal of the American College of Cardiology, vol. 50, no. 22, pp. 2173-2195, 2007.

[19] E. R. DeLong, D. M. DeLong, and D. L. Clarke-Pearson, "Comparing the areas under two or more correlated receiver operating characteristic curves: a nonparametric approach," Biometrics, vol. 44, no. 3, pp. 837-845, 1988.

[20] M. J. Pencina, R. B. D’Agostino Sr., R. B. D’Agostino Jr., and R. S. Vasan, "Evaluating the added predictive ability of a new marker: from area under the ROC curve to reclassification and beyond," Statistics in Medicine, vol. 27, no. 2, pp. 157-172, 2008.

[21] J. J. Wykrzykowska, S. Garg, C. Girasis et al., "Value of the SYNTAX score for risk assessment in the all-comers population of the randomized multicenter LEADERS (Limus Eluted from A Durable versus ERodable Stent coating) trial," Journal of the American College of Cardiology, vol. 56, no. 4, pp. 272-277, 2010.

[22] C. Girasis, S. Garg, L. Raber et al., "SYNTAX score and Clinical SYNTAX score as predictors of very long-term clinical outcomes in patients undergoing percutaneous coronary interventions: a substudy of SIRolimus-eluting stent compared with pacliTAXel- eluting stent for coronary revascularization (SIRTAX) trial," European Heart Journal, vol. 32, no. 24, pp. 3115-3127, 2011.

[23] T. Palmerini, P. Genereux, A. Caixeta et al., "Prognostic value of the SYNTAX score in patients with acute coronary syndromes undergoing percutaneous coronary intervention: analysis from the ACUITY (Acute Catheterization and Urgent Intervention Triage StrategY) trial," Journal of the American College of Cardiology, vol. 57, pp. 2389-2397, 2011.

[24] V. Farooq, Y. Vergouwe, P. Genereux et al., "Prediction of 1year mortality in patients with acute coronary syndromes undergoing percutaneous coronary intervention: validation of the logistic clinical SYNTAX (Synergy Between Percutaneous Coronary Interventions With Taxus and Cardiac Surgery) score," JACC cardiovascular Interventions, vol. 6, pp. 737-745, 2013.

[25] S. Garg, G. Sarno, P. W. Serruys et al., "Prediction of 1-year clinical outcomes using the SYNTAX score in patients with acute ST-segment elevation myocardial infarction undergoing 
primary percutaneous coronary intervention: a substudy of the STRATEGY (Single High-Dose Bolus Tirofiban and Sirolimus-Eluting Stent Versus Abciximab and Bare-Metal Stent in Acute Myocardial Infarction) and MULTISTRATEGY (Multicenter Evaluation of Single High-Dose Bolus Tirofiban Versus Abciximab With Sirolimus-Eluting Stent or Bare-Metal Stent in Acute Myocardial Infarction Study) trials," JACC Cardiovascular Interventions, vol. 4, no. 1, pp. 66-75, 2011.

[26] T. Akgun, V. Oduncu, A. Bitigen et al., "Baseline SYNTAX Score and long-term outcome in patients with ST-segment elevation myocardial infarction undergoing primary percutaneous coronary intervention," Clinical and Applied Thrombosis, vol. 21, no. 8, pp. 712-719, 2014.

[27] V. Farooq, P. W. Serruys, C. Bourantas et al., "Incidence and multivariable correlates of long-term mortality in patients treated with surgical or percutaneous revascularization in the synergy between percutaneous coronary intervention with taxus and cardiac surgery (SYNTAX) trial," European Heart Journal, vol. 33, no. 24, pp. 3105-3113, 2012.

[28] S. J. Brener, A. J. Prasad, R. Abdula, and T. J Sacchi, "Relationship between the angiographically derived SYNTAX score and outcomes in high-risk patients undergoing percutaneous coronary intervention," Journal of Invasive Cardiology, vol. 23, no. 2, pp. 66-69, 2011.

[29] T. Chakravarty, M. H. Buch, H. Naik et al., "Predictive accuracy of SYNTAX score for predicting long-term outcomes of unprotected left main coronary artery revascularization," American Journal of Cardiology, vol. 107, no. 7, pp. 360-366, 2011.

[30] E. I. Lev, R. Kornowski, H. Vaknin-Assa et al., "Comparison of the predictive value of four different risk scores for outcomes of patients with ST-elevation acute myocardial infarction undergoing primary percutaneous coronary intervention," American Journal of Cardiology, vol. 102, no. 1, pp. 6-11, 2008.

[31] M. Taniwaki, G. G. Stefanini, S. Silber et al., "4-year clinical outcomes and predictors of repeat revascularization in patients treated with new-generation drug- eluting stents: a report from the RESOLUTE All- Comers trial (A Randomized Comparison of a Zotarolimus-Eluting Stent With an Everolimus-Eluting Stent for Percutaneous Coronary Intervention)," Journal of the American College of Cardiology, vol. 63, no. 16, pp. 1617-1625, 2014.

[32] E. P. Navarese, M. Kolodziejczak, D. J. Kereiakes, U. S. Tantry, C. O'Connor, and P. A. Gurbel, "Proprotein convertase subtilisin/kexin Type 9 monoclonal antibodies for acute coronary syndrome: a narrative review," Annals of Internal Medicine, vol. 164, no. 9, pp. 600-607, 2016.

[33] M. S. Sabatine, R. P. Giugliano, S. D. Wiviott et al., "Efficacy and safety of evolocumab in reducing lipids and cardiovascular events," New England Journal of Medicine, vol. 372, no. 16, pp. 1500-1509, 2015.

[34] J. B. Muhlestein, J. L. Anderson, B. D. Horne et al., "Effect of fasting glucose levels on mortality rate in patients with and without diabetes mellitus and coronary artery disease undergoing percutaneous coronary intervention," American Heart Journal, vol. 146, no. 2, pp. 351-358, 2003.

[35] R. R. Giraldez, R. M. Clare, R. D. Lopes et al., "Prevalence and clinical outcomes of undiagnosed diabetes mellitus and prediabetes among patients with high-risk non-ST- segment elevation acute coronary syndrome," American Heart Journal, vol. 165, no. 6, pp. 918.e2-925 e2, 2013.
[36] S. Savonitto, N. Morici, C. Cavallini et al., "One-year mortality in elderly adults with non-ST-elevation acute coronary syndrome: effect of diabetic status and admission hyperglycemia," Journal of the American Geriatrics Society, vol. 62, no. 7, pp. 1297-1303, 2014.

[37] P. Deedwania, M. Kosiborod, E. Barrett et al., "Hyperglycemia and acute coronary syndrome: a scientific statement from the American Heart Association Diabetes Committee of the Council on Nutrition, Physical Activity, and Metabolism," Circulation, vol. 117, no. 12, pp. 1610-1619, 2008.

[38] M. C. Morice, P. W. Serruys, A. P. Kappetein et al., "Outcomes in patients with de novo left main disease treated with either percutaneous coronary intervention using paclitaxeleluting stents or coronary artery bypass graft treatment in the Synergy Between Percutaneous Coronary Intervention with TAXUS and Cardiac Surgery (SYNTAX) trial," Circulation, vol. 121, no. 24, pp. 2645-2653, 2010.

[39] A. P. Kappetein, T. E. Feldman, M. J. Mack et al., "Comparison of coronary bypass surgery with drug-eluting stenting for the treatment of left main and/or three-vessel disease: 3year follow-up of the SYNTAX trial," European Heart Journal, vol. 32, no. 17, pp. 2125-2134, 2011.

[40] S. J. Park, Y. H. Kim, D. W. Park et al., "Randomized trial of stents versus bypass surgery for left main coronary artery disease," New England Journal of Medicine, vol. 364, no. 18, pp. 1718-1727, 2011.

[41] D. Capodanno, G. W. Stone, M. C. Morice, T. A. Bass, and C. Tamburino, "Percutaneous coronary intervention versus coronary artery bypass graft surgery in left main coronary artery disease: a meta-analysis of randomized clinical data," Journal of the American College of Cardiology, vol. 58, no. 14, pp. 1426-1432, 2011.

[42] V. Farooq, Y. Vergouwe, L. Raber et al., "Combined anatomical and clinical factors for the long-term risk stratification of patients undergoing percutaneous coronary intervention: the Logistic Clinical SYNTAX score," European Heart Journal, vol. 33, no. 24, pp. 3098-3104, 2012.

[43] S. Garg, G. Sarno, H. M. Garcia-Garcia et al., "A new tool for the risk stratification of patients with complex coronary artery disease: the clinical SYNTAX score," Circulation Cardiovascular Interventions, vol. 3, no. 4, pp. 317-326, 2010.

[44] S. L. Chen, J. P. Chen, G. Mintz et al., "Comparison between the NERS (new risk stratification) score and the SYNTAX (synergy between percutaneous coronary intervention with taxus and cardiac surgery) score in outcome prediction for unprotected left main stenting," JACC Cardiovascular Interventions, vol. 3, no. 6, pp. 632-641, 2010.

[45] V. Farooq, S. Brugaletta, P. W. Serruys et al., "Contemporary and evolving risk scoring algorithms for percutaneous coronary intervention," Heart, vol. 97, no. 23, pp. 1902-1913, 2011.

[46] D. Capodanno, M. Miano, G. Cincotta et al., "EuroSCORE refines the predictive ability of SYNTAX score in patients undergoing left main percutaneous coronary intervention," American Heart Journal, vol. 159, no. 1, pp. 103-109, 2010.

[47] D. N. Feldman, C. L. Gade, A. J. Slotwiner et al., "Comparison of outcomes of percutaneous coronary interventions in patients of three age groups ( $<60,60$ to 80 , and $>80$ years) (from the New York State Angioplasty Registry)," American Journal of Cardiology, vol. 98, no. 10, pp. 1334-1339, 2006. 


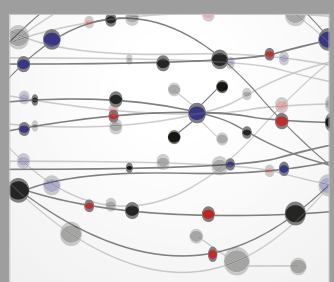

The Scientific World Journal
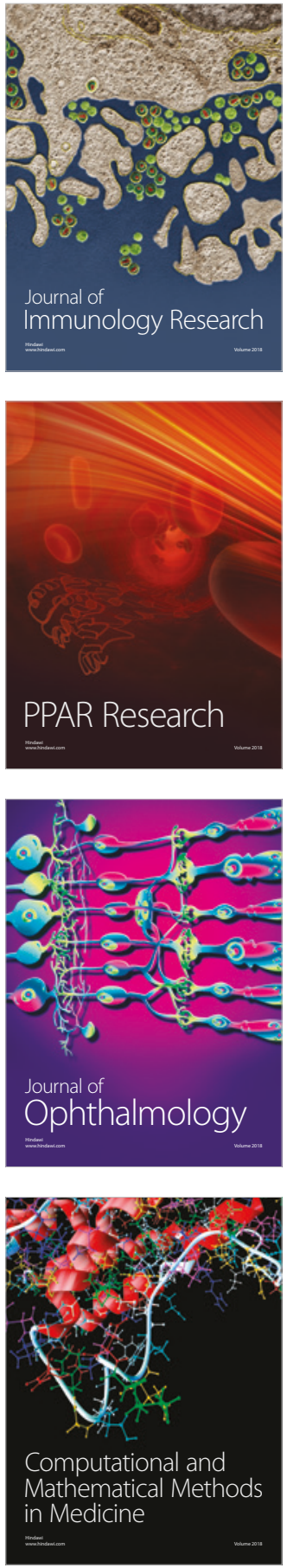

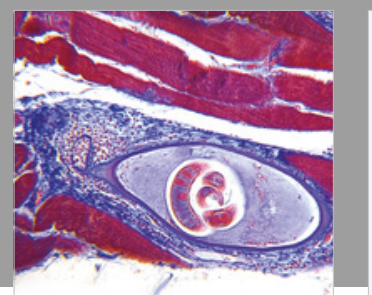

Gastroenterology Research and Practice

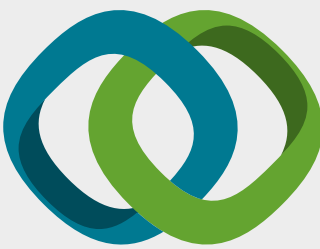

\section{Hindawi}

Submit your manuscripts at

www.hindawi.com
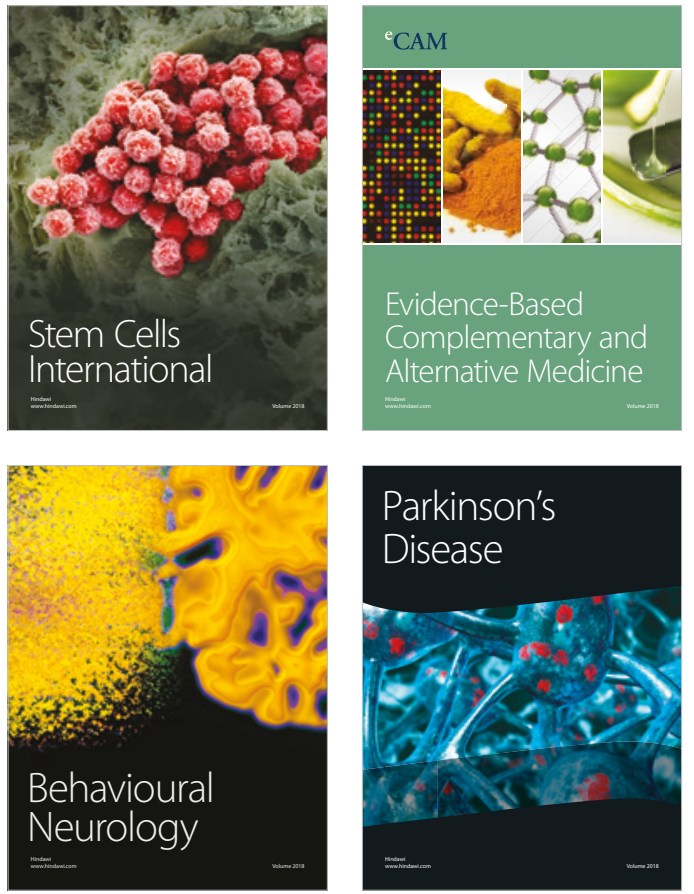

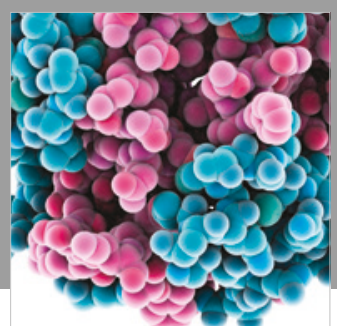

ournal of

Diabetes Research

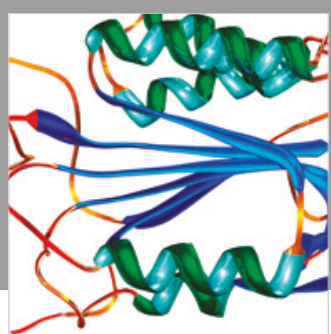

Disease Markers
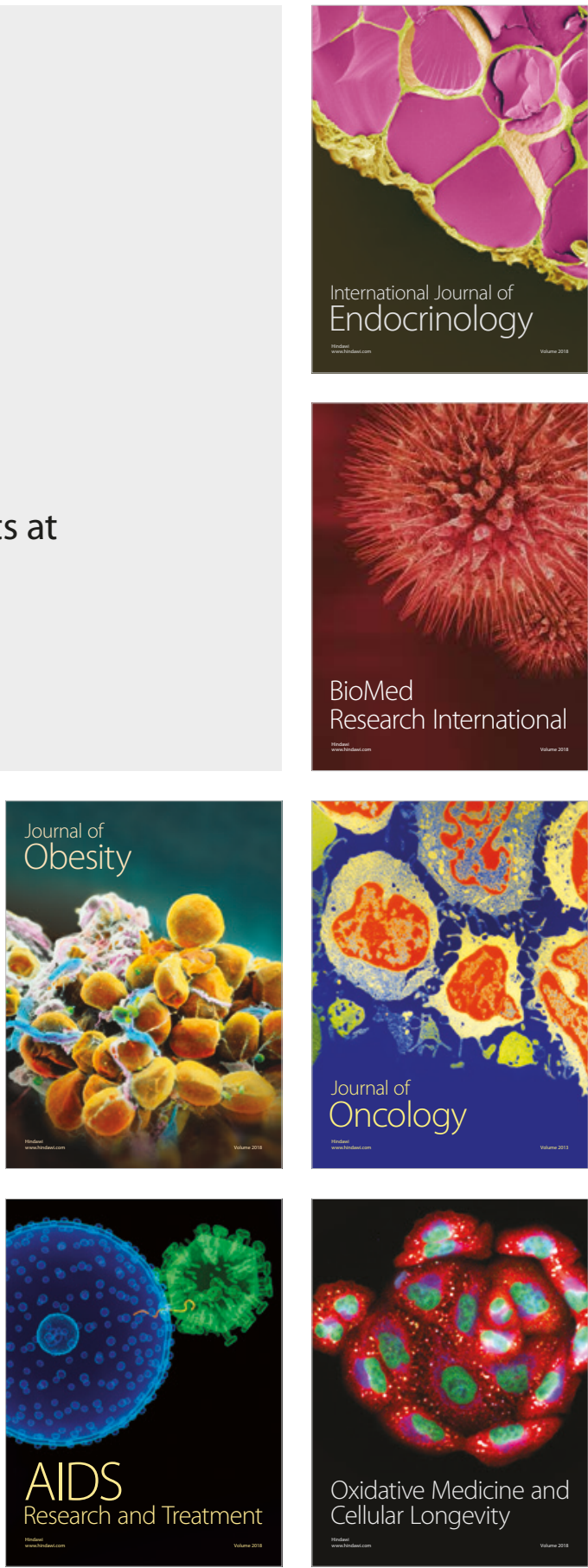